\title{
APPROXIMATION THEOREMS FOR SEMI-GROUP OPERATORS IN INTERMEDIATE SPACES
}

BY HUBERT BERENS AND P. L. BUTZER

Communicated by E. E. Hewitt, April 8, 1964

Let $X$ be a real or complex Banach-space; if $f \in X,\|f\|$ denotes the norm of $f$. If $E(X)$ denotes the Banach-algebra of endomorphisms of $X,\{T(t)\}$ is called a one-parameter semi-group in $E(X)$ of class $\left(C_{0}\right)$, if (i) $T(t) \in E(X)$ for $t \in[0, \infty), T(0)=I$ (identity); (ii) $T(t+u)$ $=T(t) T(u)$ for $t, u \in[0, \infty)$; (iii) $\lim _{t \downarrow 0}\|T(t) f-f\|=0$ for all $f \in X$.

Under these hypotheses the infinitesimal operator of $\{T(t)\}$ is a closed linear operator $A$ defined by

$$
\lim _{i \downarrow 0}\left\|t^{-1}[T(t) f-f]-A f\right\|=0
$$

with domain of definition $D(A)$ dense in $X . D(A)$ becomes a Banachspace, if the norm is defined by $\|f\|+\|A f\|$ (see E. Hille and R. S. Phillips [3, Chapter X]).

One of the authors [1] has studied the problems of best approximation in semi-group theory. Thus:

Let $\{T(t)\}$ be a semi-group of class $\left(C_{0}\right)$ defined on $X$.

(i) If $\|T(t) f-f\|=o(t)(t \downarrow 0)$, then $A f=\Theta$ and $T(t) f \equiv f$.

(ii) For each $f \in D(A)$ we have $\|T(t) f-f\|=O(t)(t \downarrow 0)$.

(iii) If $X$ is reflexive and $\|T(t) f-f\|=O(t)(t \downarrow 0)$, then $f \in D(A)$.

The statements (i) and (ii) go back to E. Hille [3, Chapter X]. For a generalization of this theorem see the cited paper as well as $\mathrm{K}$. de Leeuw [4] and P. L. Butzer and H. G. Tillmann [2].

It is the object of this note to characterize the set of elements $f$, for which the order of approximation of $f$ by $T(t) f$ is not the best possible, i.e., we will not treat saturation problems. In this case, the following general theorem holds.

Theorem 1. Let $\{T(t)\}$ be a semi-group of class $\left(C_{0}\right)$, let $T(t)[X]$ $\subset D(A)$ for each $t>0$ and $\|A T(t)\| \leqq M_{0} t^{-1}$, then

$$
\|T(t) f-f\|=O[\phi(1 / t)]
$$

implies

$$
\|A T(t) f\| \leqq M_{1}+M_{2} t^{-1} \phi(1 / t)+M_{3} \int_{1}^{1 / t} \phi(u) d u \quad(0<t \leqq 1),
$$


where $\phi(u)$ is a positive nonincreasing function in $[1, \infty)$ and $M_{i}$ $(i=0,1,2,3)$ are constants. ${ }^{1}$

We may remark that $M$. Zamansky [7] has established a theorem of this type for trigonometric polynomials.

COROLlARY. Under the conditions of Theorem 1 we have

(i) $\|T(t) f-f\|=O\left(t^{\alpha}\right) \quad(0<\alpha<1 ; t \downarrow 0)$ if and only if $\|A T(t) f\|$ $=O\left(t^{\alpha-1}\right)(t \downarrow 0)$;

(ii) if $\|T(t) f-f\|=O(t)(t \downarrow 0)$, then $\|A T(t) f\|=O(\log 1 / t)(t \downarrow 0)$.

The corollary is an immediate consequence of the theorem.

Sketch of proof of Theorem 1. Setting $t_{k}=1 / 2^{k}(k=0,1,2, \cdots)$, we denote by $U_{k}$ the operator $T\left(t_{k}\right)-T\left(t_{k-1}\right)$. Then by the semi-group property $A U_{k} f=A T\left(t_{k}\right)\left[f-T\left(t_{k-1}\right) f\right]-A T\left(t_{k-1}\right)\left[f-T\left(t_{k}\right) f\right]$, and making use of the assumptions one has

$$
\begin{array}{rlrl}
\left\|A U_{k} f\right\| & \leqq\left\|A T\left(t_{k}\right)\right\|\left\|f-T\left(t_{k-1}\right) f\right\|+\left\|A T\left(t_{k-1}\right)\right\|\left\|f-T\left(t_{k}\right) f\right\| \\
& \leqq M 2^{k-1} \phi\left(2^{k-1}\right) & (k=1,2, \cdots) .
\end{array}
$$

Now, let $t$ be given in $(0,1]$, we choose an integer $n$ such that $t_{n}<t \leqq t_{n-1}$. Then

$$
\left\|A T\left(t_{n}\right) f-A T\left(t_{n_{0}}\right) f\right\| \leqq \sum_{k=n_{0}+1}^{n}\left\|A U_{k} f\right\| \leqq 2 M \int_{2 n_{0}-1}^{1 / t} \phi(u) d u .
$$

Similarly, we get

$$
\left\|A T(t) f-A T\left(t_{n}\right) f\right\| \leqq M t^{-1} \phi(1 / t),
$$

and, furthermore,

$$
\begin{aligned}
\|A T(t) f\| \leqq & \left\|A T\left(t_{n_{0}}\right) f\right\|+\left\|A T\left(t_{n}\right) f-A T\left(t_{n_{0}}\right) f\right\| \\
& +\left\|A T(t) f-A T\left(t_{n}\right) f\right\|,
\end{aligned}
$$

which proves the theorem for $n_{0}=1$.

As an application we will discuss the singular integral of AbelPoisson. Let $f$ be a continuous, $2 \pi$-periodic function $\left(f \in C_{2 \pi}\right)$, with $\|f\|=\max _{x}|f(x)|$. Abel's method of summation of the Fourier series of $f$ defines the singular integral

$$
\begin{array}{rlrl}
{[V(t) f](x)} & =V\left(f ; e^{-t} ; x\right)=a_{0} / 2+\sum_{k=1}^{\infty}\left(a_{k} \cos k x+b_{k} \sin k x\right) e^{-k t} \\
& =\frac{1}{\pi} \int_{-\pi}^{\pi} f(u) P\left(e^{-t} ; x-u\right) d u & (0<t<\infty),
\end{array}
$$

${ }^{1}$ If $T(t)[X] \subset D(A)$ for each $t>0$, then $A T(t)$ exists as a bounded linear operator on $X$ for $t>0$; $\|A T(t)\|$ denotes the operator norm. 
with

$$
P(r ; u)=\frac{1}{2} \frac{1-r^{2}}{1-2 r \cos u+r^{2}} \quad\left(r=e^{-t} ; 0 \leqq r<1\right) .
$$

$V(t)$ is a semi-group of class $\left(C_{0}\right)$ with $\|V(t)\|=1 . D(A)$ is the set of functions $f$, for which the derivative of the conjugate function, thus $\tilde{f}^{\prime} \sim \sum_{k=1}^{\infty} k\left(a_{k} \cos k x+b_{k} \sin k x\right)$ is an element of $C_{2 \pi}$. Furthermore, $V(t)\left[C_{2 \pi}\right] \subset D(A)$ for all $t>0$ and

$$
\|A V(t)\|=\frac{1}{\pi} \int_{-\pi}^{\pi}\left|Q^{\prime}\left(e^{-t} ; u\right)\right| d u \leqq 4 t^{-1} \quad(0<t<\infty),
$$

whereby

$$
Q(r ; u)=\frac{r \sin u}{1-2 r \cos u+r^{2}} .
$$

Now, with the aid of the corollary we have

Theorem 2. Let $f \in C_{2 \pi}$, and let $V(f ; r ; x)$ be the Abel-Poisson integral. The following statements are equivalent if $0<\alpha<1$ :

(i) $\|f(x+h)-f(x)\|=O(|h| \alpha)(h \rightarrow 0)$;

(ii) $\|f(x+h)-2 f(x)+f(x-h)\|=O\left(|h|^{\alpha}\right)(h \rightarrow 0)$;

(iii) $\left\|\tilde{V}^{\prime}(f ; r ; x)\right\|=O(1-r)^{\alpha-1}(r \uparrow 1)$;

(iv) $\left\|V^{\prime \prime}(f ; r ; x)\right\|=O(1-r)^{\alpha-2}(r \uparrow 1)$;

(v) $\|V(f ; r ; x)-f(x)\|=O(1-r)^{\alpha}(r \uparrow 1)$.

The equivalence of the statements (i)-(iv) above is known, the results being mainly due to $\mathrm{G}$. $\mathrm{H}$. Hardy and J. E. Littlewood (see A. Zygmund [8, Chapter VII]). These proofs, in contrast to ours, used complex methods. The fact that (v) is equivalent to (i) is a new contribution.

In some of his papers, J. L. Lions [5] has studied trace theorems and theorems of interpolation in semi-group theory. He introduced the so-called intermediate spaces $X[p, \alpha, A]$ : Let $\{T(t)\}$ be a semigroup of class $\left(C_{0}\right)$ with $\|T(t)\| \leqq M_{0}$ for all $t \geqq 0$. We denote by $X[p, \alpha, A]$ the set of elements $f \in X$ for which the integral

$$
\int_{0}^{\infty} t^{(\alpha-1) p}\|T(t) f-f\| p d t
$$

exists, where $-1 / p<\alpha<1-1 / p, 1 \leqq p \leqq \infty . X[p, \alpha, A]$ becomes a Banach-space under the norm 


$$
\|f\|+\left\{\int_{0}^{\infty} t^{(\alpha-1) p}\|T(t) f-f\|^{p} d t\right\}^{1 / p} .
$$

(If $p=\infty$, the modification is evident.) It is easy to see that

$$
D(A) \subset X[p, \alpha, A] \subset X
$$

With methods stated above we can prove the following theorem concerning $X[p, \alpha, A]$.

TheOREM 3. Let $\{T(t)\}$ be a semi-group of class $\left(C_{0}\right)$, let $\|T(t)\|$ $\leqq M_{0}, T(t)[X] \subset D(A)$ for each $t>0$ and $\|A T(t)\| \leqq M_{1} t^{-1}$, then $f \in X[p, \alpha, A]$ if and only if the integral

$$
\left\{\int_{0}^{\infty} t^{\alpha p}\|A T(t) f\|^{p} d t\right\}^{1 / p}
$$

is finite.

By use of this theorem one may infer some of the results due to M. H. Taibleson [6] for the singular integral of Poisson-Cauchy in $n$-dimensional Euclidean space, since this integral is a semi-group operator satisfying the conditions of Theorem 3 .

The proofs of these and further results will appear elsewhere.

\section{REFERENCES}

1. P. L. Butzer, Über den Grad der Approximation des Identitütsoperators durch Halbgruppen von linearen Operatoren und Anwendungen auf die Theorie der singulären Integrale, Math. Ann. 133 (1957), 410-425.

2. P. L. Butzer and H. G. Tillmann, An approximation theorem for semi-groups of operators, Bull. Amer. Math. Soc. 66 (1960), 191-193.

3. E. Hille and R. S. Phillips, Functional analysis and semi-groups, Amer. Math. Soc. Colloq. Publ. Vol. 31, rev. ed., Amer. Math. Soc., Providence, R. I., 1957.

4. K. de Leeuw, On the adjoint semi-group and some problems in the theory of approximation, Math. Z. 73 (1960), 219-234.

5. J. L. Lions, Théorèmes de trace et d'interpolation. I, Ann. Scuola Norm. Sup. Pisa 13 (1959), 389-403.

6. M. H. Taibleson, Lipschitz classes of functions and distributions in $E_{n}$, Bull. Amer. Math. Soc. 69 (1963), 487-493.

7. M. Zamansky, Classes de saturation de certains procédés d'approximation des séries de Fourier des fonctions continues et applications a quelques problèmes d'approximation, Ann. Sci. Ecole Norm. Sup. (3) 66 (1949), 19-93.

8. A. Zygmund, Trigonometrical series, Vols. I, II, rev. ed., Cambridge Univ. Press, Cambridge, 1959.

The Technical University of Aachen, Aachen, Germany 